\title{
Introduction to the Special Issue "The 2009 WE-Heraeus-Seminar on satellite remote sensing of aerosols: techniques, limitations, and validation"
}

\author{
A. Kokhanovsky ${ }^{1}$ and S. Kinne ${ }^{2}$ \\ ${ }^{1}$ Institute of Environmental Physics, University of Bremen, O. Hahn Allee 1, 28334 Bremen, Germany \\ ${ }^{2}$ Max Planck Institute for Meteorology, Hamburg, Germany
}

Atmospheric air contains appreciable numbers of small solid and liquid aerosol particles, which influence climate, visibility, planetary albedo, cloud lifetime, and also atmospheric pollution. The general distribution of atmospheric aerosol on a planetary scale is reasonably well understood. However, the precise determination of the spatial and temporal variability of aerosol concentration, chemical composition, and microstructure remains a challenge. This task is very complex and requires complementary and coordinated efforts of many research teams involved in experimental and theoretical studies of atmospheric aerosol properties using in-situ observations, remote sensing methods, and modeling.

With their ability to provide data samples with global coverage, satellite measurements play an important role in capturing the spatial heterogeneity of atmospheric aerosol. The primary parameter retrieved from a dedicated satellite aerosol sensor is the aerosol optical thickness (AOT), which is the vertically integrated attenuation of light by aerosol at a given wavelength. AOT is proportional to the columnar amount of suspended particles. However, AOT is also influenced by size, composition and shape of the aerosol particles.

Retrievals of AOT from spaceborne observations require the development of inversion techniques based on measurements of characteristics of reflected sunlight such as the spectral and angular distributions of intensity. An important element of these retrievals is the selection of the appropriate aerosol model (in terms of composition size and shape) and the removal of surface contributions, which contaminate the aerosol signal. Thus, sensors with multi-spectral, multiviewing and, especially, polarization capability are preferred in order to reduce the need for a-priori assumptions. In addition, active remote sensing by lidar from space provides valuable insights into aerosol vertical distribution. Thus, space deployments of spectro-photopolarimeters (as on POLDER or on the failed GLORY mission) and of lidar systems (as on CALIPSO) are essential elements for improved characterizations of atmospheric aerosol.

The application of spaceborne polarimetry, passive spectro-radiometers (single-view, dual-view and multiangular) and lidars for the determination of aerosol optical properties from space was the central theme at the 439th WEHeraeus-Seminar held at the Bad Honnef Physics Center (Germany, 17-19 August 2009). 70 scientists from 16 countries took part in the discussion of relevant scientific issues. This special issue of AMT summarizes selected presentations of the meeting. More information about the meeting is available via the web: http://www.iup.uni-bremen.de/ $\sim$ hoyning/. We are grateful to Wilhelm and Else HerauesStiftung for the support of the workshop.

The issue is dedicated to the memory of Chieko Kittaka (1964-2009), a colleague and collaborator of many of the workshop participants. Her enthusiasm, analytic skills, and love of science were an inspiration to us all. She is truly missed.

\section{Correspondence to: A. Kokhanovsky}

(alexk@iup.physik.uni-bremen.de)

Published by Copernicus Publications on behalf of the European Geosciences Union. 